\title{
HLA-B27-related pathway mediates syndesmophyte formation in AS
}

Ankylosing spondylitis (AS) is characterized by the formation of syndesmophytes and occurs more frequently in HLA-B27+ individuals, but whether these two phenomena are related has been uncertain. According to the results of a new study, HLA-B27 misfolding is directly linked to syndesmophyte formation via tissue-nonspecific alkaline phosphatase (TNAP), in a process that can be targeted therapeutically.

"We derived mesenchymal stem cells (MSCs) from the entheses of patients with AS to address if there is any abnormality in AS-derived MSCs," explains co-corresponding author Kuo-I Lin. "We did not find any significant differences in the production of the cytokines or chemokines that we tested in AS-derived MSCs as compared with control MSCs, but AS-derived MSCs did show accelerated mineralization upon osteogenic induction."
The researchers were surprised to discover that this enhanced mineralization was not regulated by the transcription factor RUNX2, which is usually associated with this process, but was instead regulated by TNAP via the transcription factor RAR $\beta$. Further in vitro investigations revealed that unfolded HLA-B27 causes the upregulation of TNAP in AS-derived MSCs via the IRE1 $\alpha$ sXBP1 pathway of the unfolded protein response. Crucially, serum concentrations of bone-specific TNAP were increased in patients with AS compared with healthy individuals from two different cohorts and correlated with disease progression.

"We also implanted AS-derived MSCs near the mechanically eroded lumber spine of NOD-SCID mice to test whether they show enhanced bone formation in vivo," says co-corresponding author

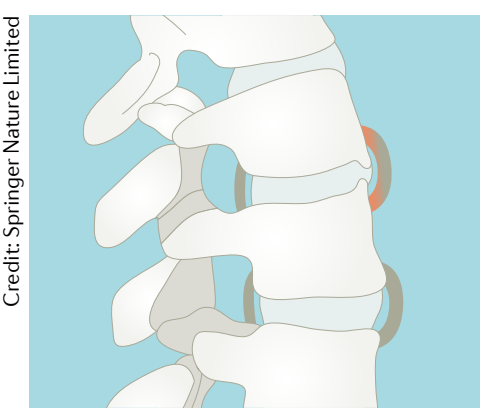

causes the upregulation of TNAP in AS-derived MSCs

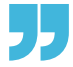

\section{OSTEOARTHRITIS}

\section{Inhibit tankyrase to preserve OA cartilage?}

A new study published in Nature Communications identifies tankyrase, a poly-ADP-ribosyltransferase, as a regulator of cartilage anabolism and suggests that suppression of tankyrase activity contributes to restoration of cartilage matrix. The research could inform the development of new therapies for osteoarthritis (OA).

"OA development has been primarily associated with increased catabolic gene expression, such as genes encoding matrix-degrading enzymes. We think that our study suggests that stimulating the anabolic axis could serve as an approach to counter cartilage loss in OA and possibly to regenerate cartilage," says corresponding author Jin-Hong Kim.

Tankyrase, encoded by TNKS or TNKS2, was previously known to be involved in various processes including WNT signalling and telomere maintenance. In the present study, transcriptome analysis in numerous strains of BXD mice revealed that tankyrase gene expression negatively correlated with expression of cartilage matrix genes. Knockdown of both Tnks and Tnks2 or treatment with tankyrase inhibitors induced the expression of cartilage matrix genes in mouse chondrocytes, suggesting that tankyrase inhibition promotes pro-anabolic pathways in cartilage.

The researchers also demonstrated that in human cartilage affected by OA, tankyrase expression is upregulated and inversely correlated with expression levels of type II collagen and aggrecan, compared with unaffected tissue. Consistent with these findings, expression of tankyrase in mouse knee joints was increased and that of aggrecan and the transcription factor SOX9 (a master regulator of chondrogenesis) decreased 8 weeks after surgery to induce post-traumatic $\mathrm{OA}$.
Shih-Chieh Hung. "This new animal model seems to mimic the development of new bony appositions in patients."

Interestingly, treatment of these mice with the TNAP inhibitors levamisole (an anti-helminthic drug) or pamidronate (a bisphosphonate) substantially reduced syndesmophyte formation. "In the future, we hope to identify new drug candidates, as currently available TNAP inhibitors may have some adverse effects," concludes Lin.

Joanna Clarke

ORIGINAL ARTICLE Liu, C.-H. et al. HLA-B27mediated activation of TNAP phosphatase promotes pathogenic syndesmophyte formation in ankylosing spondylitis. J. Clin. Invest. https:// doi.org/10.1172/JCl125212 (2019)
In mouse chondrocytes, tankyrase inhibition inverted the expression patterns of $\mathrm{OA}$-associated genes. In vivo, hydrogel-mediated intraarticular delivery of tankyrase inhibitors to the knees of mice with surgically induced $O A$ protected against OA progression. This protective effect was associated with increased expression of cartilage matrix constituents, reduced production of catabolic regulators and preserved expression of SOX9 compared with vehicle administration only.

Tankyrase inhibition also enhanced the chondrogenic differentiation of human bone-marrow-derived mesenchymal stem cells and improved the regeneration of articular cartilage lesions.

Together, the findings could have implications for the development of therapies for $\mathrm{OA}$ aimed at cartilage regeneration.

\section{Sarah Onuora}

ORIGINAL ARTICLE Kim, S. et al. Tankyrase inhibition preserves osteoarthritic cartilage by coordinating cartilage matrix anabolism via effects on SOX9 PARylation. Nat. Commun. 10, 4898 (2019) 